Highly Energetic Physical Processes and

Mechanisms for Emission from Astrophysical Plasmas

IAU Symposium, Vol. 195, 2000

P. C. H. Martens, S. Tsuruta, and M. A. Weber, eds.

\title{
Hidden Blazars In Radio Loud Quasars?
}

F. Ma

The University of Texas at Austin, Austin, TX 78712, U.S.A.

\begin{abstract}
We present the initial results from our search for hidden blazars via CIV emission line variability among radio loud quasars. The implications on models of quasar Broad Emission Line Regions are discussed, and we focus on the tidally disrupted star model.
\end{abstract}

Beamed emission associated with jets in various astrophysical locations are often not observable if the beam is pointing away from the observers. The more isotropic reprocessed emission from interactions between the beam and ambient gas may offer indirect evidence for existence of the beam. One example is beamed synchrotron radiation, presumably existing in every radio-loud quasar but not in radio-quiet AGN, which can heat the BELR (Broad Emission Line Region) gases, greatly enhancing the CIV line emission (Ma \& Wills 1998). We expect to see CIV line variability not associated with Ly $\alpha$ or CIII line variations in every radio loud quasar. A sample of 30 " $\mathrm{z} \sim 2$ " radio loud quasars is being observed at McDonald Observatory to search for "hidden blazars". Our most interesting candidate so far is $0106+01$. The EWs (equivalent widths) of Ly $\alpha /$ CIV are $275 \AA / 193 \AA$ (1979); $291 \AA / 119 \AA$ (1987); and $328 \AA / 118 \AA$ (1998), respectively.

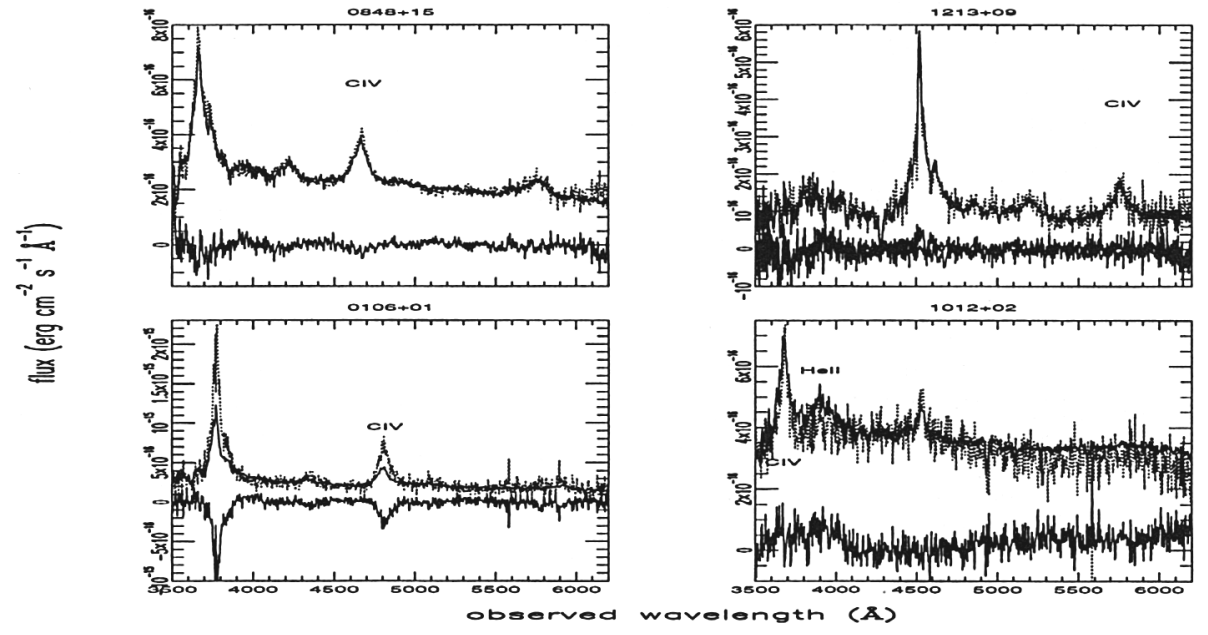

Figure 1. New spectra (solid lines) are compared with historical data taken $\sim 10$ years ago. The EW of CIV does not change in $0106+01$ in 1987 and 1998. 1012+02 shows interesting He II line variation.

If the disk-wind model is ruled out by our observations, very few choices for the origin of BELR gases are left. We study a BELR model of tidally disrupted 
stars (c.f., Shields 1989; Roos 1992) with winds blown off the tidal streams by the radiation pressure. Most of the BELR parameters are determined by the central black hole mass and the disruption process. The examples of line profiles in Figure 2 shows that this model can reproduce, in general, the observed line profiles. The winds from the tidal streams have a covering factor comparable to that of the tidal streams, lowering the requirement of a high tidal disruption rate. The winds and the tidal streams have very distinct densities $\left(10^{6-8} \mathrm{~cm}^{-3}\right.$ vs. $10^{10-13} \mathrm{~cm}^{-3}$ ) at similar spatial locations. The winds also have higher velocities.

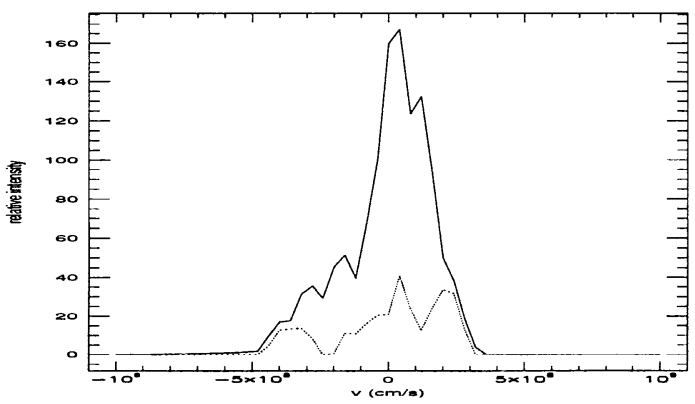

Figure 2. Civ emission line profile from the tidal remnants (solid line) and winds (dotted line) of 11 tidally disrupted stars.

Our observations could offer a method to observe reprocessed beamed emission. This idea may also apply to the observations of Gamma-Ray Bursts. If the tidal streams/winds model for BELR is correct, we could outline such a picture of quasar-galaxy connection: in normal galaxies, the tidal disruption rate is lower than $10^{-4} \mathrm{yr}^{-1}$; it is boosted to $\sim 1 \mathrm{yr}^{-1}$ due to galaxy mergers and triggering AGN activities. The neutrino flux associated with the AGN activities may heat the stars from their cores (MacDonald, Stanev, \& Biermann 1991) and modify the stellar population around the massive black hole, e.g., the stars might be mostly giants rather than main sequence stars; the giants can still be disrupted after the the black hole grows so massive that it will swallow main sequence stars whole. The AGN activities die out once (1) the tidal disruption rate drops back to the slow diffusion process without the perturbation of the gravitational potential, or (2) the central black hole grows over $10^{9} M_{\odot}$, since it will then swallow giants whole rather than disrupt them.

Acknowledgments. I thank Bev Wills for guidance and help with the observations, Greg Shields for critical comments on the whole project, Paul Francis for making some of his spectra available in digital form, and Gary Ferland for making his code CLOUDY available to the public.

\section{References}

Ma, F., \& Wills, B. J. 1998, ApJ, 504, L65

MacDonald, J., Stanev, T., \& Biermann, P. L. 1991, ApJ, 378, 30

Roos, N. 1992, ApJ, 385, 108

Shields, G. A. 1989, in IAU Symp. 134, Active Galactic Nuclei, eds. D. E. Osterbrock \& J. S. Miller (Dordrecht: Kluwer), 577 